\title{
Migration and Myelination by Adult Glial Cells: Reconstructive Analysis of Tissue Culture Experiments
}

\author{
Merrill K. Wolf, ${ }^{\star}$ Monty C. Brandenberg, $\dagger$ and Susan Billings-Gagliardi ${ }^{\star}$ \\ *Department of Anatomy, University of Massachusetts Medical School, Worcester, Massachusetts 01605, and \\ †lmage Graphics Laboratory, Department of Neuroscience, Children's Hospital Medical Center, Boston, \\ Massachusetts 02115
}

\begin{abstract}
Adult glia are capable of at least limited myelination of CNS axons. However, it is difficult to quantitate their myelination or migratory capacities and to examine contributions of the CNS environment or exogenous factors that might promote or inhibit this process in situ. We have therefore developed a mouse tissue culture system in which optic nerve glia (in the form of appropriately handled optic nerve) are added to chemically demyelinated cerebellar axons. Optic nerve up to postnatal day 411 (P411) contains cells that can migrate out of the nerve into the cerebellar explant and form myelin around its axons. The success rate for myelin formation in these cultures is $\mathbf{5 7 \%}$ for immature (P7-11) glia and 55\% for adult (P50-411) glia. Computer-generated reconstructions of cultures containing immature (P8) and adult ( $P 89$ and P139) nerves demonstrate that in all 3 cases the glia may migrate more than $0.6 \mathrm{~mm}$ before myelinating axons, assuming the shortest possible track. Both the age limit for myelination and distance limit for migration, if any, remain to be determined for these adult glia. In successful cultures, myelin always directly abuts the optic nerve surface, whether or not it also extends further, suggesting that migrating glia may depend upon contact guidance by myelin-receptive axons. We conclude that this culture system is a useful model of adult CNS myelin regeneration, in which one can examine the influence of potential trophic or toxic factors on specific aspects of myelinating glial cell behavior.
\end{abstract}

For studies of hypomyelinated mutant mice, we have made extensive use of an organotypic culture system in which a severed optic nerve from an immature mouse is explanted in direct contact with cerebellar tissue previously explanted from another immature mouse (Wolf et al., 1981). The optic nerve, of course, contains myelinating glial cells but no viable axons, while the cerebellum contains receptive axons but lacks normal myelinating glia of its own either because of a mutant disorder (BillingsGagliardi et al., 1983, 1984; Wolf et al., 1981) or because of treatment with mitotic inhibitors (Stanhope et al., 1986). In this culture system, cells originating in the optic nerve leave the nerve, migrate for considerable distances into the foreign neuropil of the cerebellum, and myelinate cerebellar axons that they

\footnotetext{
Received Feb. 18, 1986; revised May 27, 1986; accepted June 12, 1986.

We thank Marsha E. Pomeroy, Gail B. Stanhope, and Mary V. Gray for their valued collaboration and assistance with the culture system, and Robert A. Pearlstein and Richard L. Sidman for their generous advice and assistance with 3-dimensional reconstruction. Color photography by Joseph A. Gagliardi. Supported by National Institutes of Health Grant NS 11425 (Javits Award) and a research contract from the Stifel Paralysis Research Foundation to M.K.W. and S.B-G., and Grant NS 20822 to Richard L. Sidman, Head, Department of Neuroscience, Children's Hospital Medical Center.

Correspondence should be addressed to Merrill K. Wolf, Department of Anatomy, University of Massachusetts Medical School, 55 Lake Avenue North, Worcester, MA 01605

Copyright $(\overline{\mathcal{C}}) 1986$ Society for Neuroscience $0270-6474 / 86 / 123731-08 \$ 02.00 / 0$
}

would never encounter during normal development (BillingsGagliardi et al., 1983). Experiments using this system provided much key evidence about the mutant disorders by permitting confrontations between myelinating glial cells and axons of different genotypes. They also suggested that the system could potentially be an important tool for investigating the capacity of mammalian CNS to regenerate myelin after injury or disease. The apparent limitation of the system, suggested by our previous experiments, was that myelin was formed only by optic nerve glia explanted during the first 14 postnatal days (P0-14), before or during the period of active myelinogenesis (Kelton and Rauch, 1962; Skoff et al., 1976). Similarly, the experiments by Lachapelle et al. (1984) demonstrated long-distance migration of myelinating glial cells grafted into myelin-deficient host brains. However, these investigations, like our previous culture studies, were performed with newborn donors and hosts. Myelin formation by immature optic nerve glia, whether in vitro or in situ, would more likely correspond to normal initial myelin development, rather than to myelin regeneration at a later time.

We now have found a series of technical modifications of the culture system that make it possible to obtain myelin formation by optic nerve glia from mice that are not only adult, but even aging (up to P411). Computer-generated 3-dimensional (3-D) reconstructions have been employed to compare quantitative stereotactic information on the migratory and myelination capabilitics of optic ncrve glia from $P 8,89$, and 139 . A preliminary report of this work has appeared in abstract form (Wolf et al., 1985).

\section{Materials and Methods}

\section{Cultures}

The basic culture methods have been described (Schwing-Stanhope and Wolf, 1983). In brief, cerebella from P0 mice derived from B6C3F1 hybrid stocks are dissected into 8 parasagittal slabs; explanted onto collagen-coated coverslips, 2 slabs per coverslip; and fed $50 \mu \mathrm{l}$ of medium containing $50 \%$ fetal calf serum from a pretested lot, $25 \%$ Dulbecco's minimum essential medium, and $25 \%$ balanced salt solution mixed with $5 \%$ glucose and cytosine arabinoside (Ara-C) to produce final concentrations, in the complete medium, of $0.6 \%$ glucose and approximately $1.8 \times 10^{-5} \mathrm{M}$ Ara-C. This dose of Ara-C was previously shown to prevent the development of myelin and mature oligodendrocytes in cerebellar cultures (Seil et al., 1980; Stanhope et al., 1986). We now find that, for each new manufacturer's lot of the drug, the dose must be empirically adjusted to produce complete demyelination with minimum damage to nondividing cells. After $4 \mathrm{~d}$ in vitro (DIV), the cultures are refed medium with Ara-C. After 8 DIV the cultures are washed twice in balanced salt solution to remove Ara-C and fed medium containing $25 \%$ fetal calf serum, $25 \%$ saline extract of $9 \mathrm{~d}$ chicken embryos, $25 \%$ Dulbecco's, and $25 \%$ balanced saline with glucose but without Ara-C. At this point, 2 of the 4 cultures obtained from each mouse receive an additional explant of optic nerve from another mouse, ranging in age from $\mathrm{P} 7$ to $\mathrm{P} 411$ for the experiments reported here. The 
Figure 1. $\quad$ P8 optic nerve $(O N)$ plus Ara-C-treated cerebellum $(C R B M)$. The optic nerve is surrounded by a tube of meninges. At the left, the cut end of the optic nerve has fused with the cerebellar explant, obliterating any border between the two. Note the incisures in the optic nerve meninges where fusion of optic nerve and cerebellar tissue has also occurred ( $a r$ rows). When this figure is compared with Figure 3, $a$ and $b$, which are computer-aided reconstructions of this same P8 culture, myelin profiles in the cerebellar explant are associated with these incisures, as well as with the larger cut surface at the end of the optic nerve. Some of the many myelin sheaths formed around cerebellar axons in this culture by $\mathrm{P} 8$ myelinating glia are shown at higher magnification in the inset (arrowheads). Bar, $100 \mu \mathrm{m}$; inset bar, $5 \mu \mathrm{m}$.

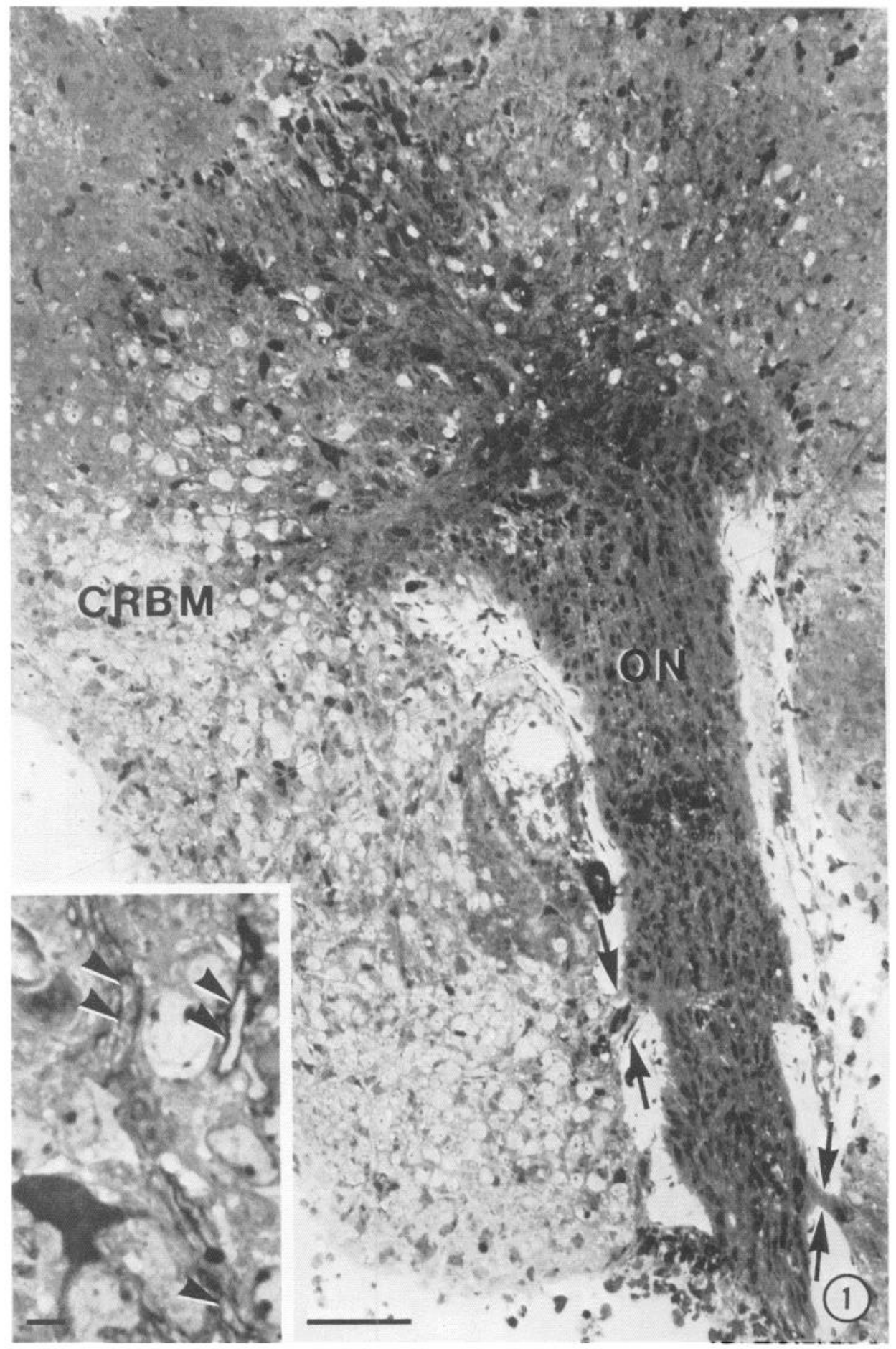

essential for the experiment to succeed. Since the meninges are impossible to remove cleanly, they are incised at several points along the length of the nerve to increase the amount of cut surface from which myelinating glial cells are free to migrate into the cerebellum.

If adult nerve is handled like immature nerve and explanted in 1 piece, the result is widespread cell death in the nerve, and usually in the cerebellar explants as well. We thought proteolytic enzymatic dissociation of the tissue might be necessary, and began preliminary experiments. However, it was found that bathing the nerve in $\mathrm{Ca}-$ and $\mathrm{Mg}$-free saline for a few minutes prior to, and then during, dissection makes the thick meninges of the adult nerve much more friable. They can then be partially removed, thus permitting the nerve to be cleanly sliced into 8-12 thick disk-shaped pieces. In the case of the oldest, and more pieces is detrimental: The small fragments are harder to place accurately in direct contact with the cerebellar tissue, a relationship 
therefore largest, nerves, these disks are halved. One or two of the resulting fragments are placed in direct contact with cerebellar tissue. No enzyme treatment or dissociation of the tissue is necessary. Among other advantages, this permits the starting point for glial migration to be reckoned from the original location of the optic nerve fragment.

\section{3-D reconstructions}

For computer reconstruction, cultures are fixed in aldehydes and osmium, embedded in Epon, serially sectioned at $2 \mu \mathrm{m}$, and stained with toluidine blue. In our first reconstruction, omitted from this report, the plane of sectioning was apparently oblique to the original plane of the culture-bearing coverslip. This led to a systematic artifact of angular distortion being introduced into the reconstruction at the stage of rcgistering adjacent-section maps. Embedding subsequent cultures in a thin $(1.0 \mathrm{~mm})$ wafer of plastic and careful orientation prior to sectioning appeared to minimize this artifact. Cultures containing optic nerve of 3 selected ages, approximately P8, 90, and 140, were serially sectioned. After examining 3-5 cultures at each age, 1 was chosen for reconstruction based on the amount and extent of myelination and on the quality of the section series.

Digitization of the culture image is carried out with the custom hardware and software of the Image Graphics Laboratory. The Zeiss Universal microscope is fitted with 3-axis stepper motor staging $(0.5 \mu \mathrm{m}$ step size in $X, Y$, and $Z$ axes) running under local microcomputer control. A camera lucida attachment allows viewing of a Megatek 7210 graphics display as a tightly registered overlay to the microscope image. This permits the user to trace features in the field via intcraction with a data tablet. The microscope graphics subsystems are coupled to a host Digital Equipment Corporation Vax 11/780 super minicomputer (Pearlstein et al., 1986). For each section, a boundary contour is drawn at low magnification; the area of the contour is then divided into a matrix of squares, each corresponding to 1 high-magnification field. The computer drives the microscope stage to successive squares in any desired sequence, and features which are clearly visible only at high magnification, in this case myelin and the meningeal coverings of the optic nerve fragment, are marked. Thus, each section is digitally represented as a map comprising a boundary contour and points representing the locations of myelin and optic nerve. The maps are then stacked and registered, using the fit of both boundary contours and object markers to guard against the angular distortion artifact.

The data file as a whole is assigncd a number for scetion thickncss, which sets the scale of the $Z$-axis. It is now ready for visual display or for the generation of numerical parameters. The visual display can be manipulated by rotation; by enhancement, suppression, or deletion of any of its features; by hidden line suppression and pseudocoloring; and by arbitrary alteration of the scale of the $Z$-axis to produce an "exploded" view that clarifies internal detail. The numerical software has been used to measure, in 3 dimensions, the distances between each piece of myelin and the nearest point of the optic nerve fragment. This provides a first approximation to the minimum distances over which glial cells can migrate to form myelin in this system.

\section{Results}

Table 1 compares our series of Ara-C-treated cultures with added adult optic nerve with a similar series of cultures with added immature optic nerve, i.e., P7-11. Ninety-five cultures with adult nerve and their controls have been examined. Twentyfour were excluded from further consideration because a small amount of myelin was detected in the controls and are omitted from Table 1 . Of the remaining 71 cultures, 39 , or $55 \%$, contained myelin formed by the optic nerve glia. In the corresponding series with $\mathrm{P} 7-11$ nerve, 24 out of 42 , or $57 \%$, contained myelin formed by the optic nerve glia. The success rate was not significantly different for optic nerves under and over P100. The success of this experiment is thus independent of the age of the nerve, at least over a wide range, when the tissue is appropriately handled. Furthermore, the light-microscopic appearance of the myelin from immature or adult donor nerve is indistinguishable (compare Figs. 1 and 2). The older glial cells do, however, require up to 6-8 d more in vitro to make their myelin. Optic nerve is added to all cultures at $8 \mathrm{DIV}$. If it is P8 nerve, myelin may be visible in 5-7 d, i.e., 13-15 DIV, and any culture that
Table 1. Number of Ara-C-treated cerebellar cultures producing myelin after addition of optic nerve

\begin{tabular}{ll} 
Age of donor optic nerve & $\begin{array}{l}\text { Cultures with myelin/total } \\
\text { cultures }\end{array}$ \\
\hline $\begin{array}{l}\text { Immature } \\
\text { P7-1 }\end{array}$ & $24 / 42(57 \%)$ \\
Adult & \\
P50-93 & $22 / 42(52 \%)$ \\
P118-146 & $13 / 19(68 \%)$ \\
P376-411 & $4 / 10(40 \%)$ \\
Total & $39 / 71(55 \%)$ \\
\hline
\end{tabular}

is going to develop any myelin does so by $18-19$ DIV. In the case of adult nerve, however, many cultures that have no visible myelin at 20 DIV develop some by 24-26 DIV. We suspect, based on our limited experience to date, that the oldest glial cells take the longest time to make the myelin in vitro.

The 3 cultures chosen for reconstruction are designated P8, 89 , and 139 after the age of added optic nerve. P8 is illustrated in the most detail, to show, step by step, the relationship between the microscopic sections and the reconstruction. Figure 1 is a low-magnification view of a single section through P8, taken at the thickest part of the optic nerve for best correspondence with the 3-D views following. The meningeal coverings of the optic nerve are clearly identifiable, but the tissue of the nerve itself cannot be clearly demarcated from the tissue of the cerebellar explant with which it has fused. The part of the section to the left of the cut end of the nerve contains numerous myelin profiles, which are illustrated in the inset.

Figure 3, $a-h$, displays reconstructions of $\mathrm{P} 8,89$, and 139 in various orientations. The boundary contour has been pseudocolored blue, the myelin green, and the optic nerve meninges red. The yellow color seen in several places results from superimposition of green myelin and red optic nerve signals on the screen. Hidden line suppression has been used in all displays. In Figure $3 a, \mathrm{P} 8$ is viewed from its top surface (facing the lying drop of medium); in Figure $3 b$, it is viewed from its bottom surface (facing the collagen), corresponding to the plane of the section in Figure 1. Myelin is extremely abundant and appears to be located at the maximum possible distance from the optic nerve. However, it is not uniformly distributed in the culture, but is confined to about half the area of the cerebellar explants. Comparing Figure $3 b$ to Figure $3 a$ shows that the cerebellar tissue tends to burrow into the collagen gel substrate, forming little indentations, or "pegs." An incision in the optic nerve, not visible from above, is clearly seen from below. Myelin profiles appear to be within the incision (actually they are directly beneath it) and are extremely numerous in adjacent parts of the cerebellum. In all cultures containing myelin, regardless of the age of the optic nerve, myelin is always found in direct apposition to a cut surface of the optic nerve fragment, from which region it may extend in a fairly continuous swath to more remote areas of the culture. We have never seen a culture containing myelin that has a distinct myelin-free zone around the optic nerve. Figure $3 c$ shows $\mathrm{P} 8$ rotated about $60^{\circ}$, while Figure $3 d$ shows $\mathrm{P} 8$ rotated $90^{\circ}$ so that each section is vicwed edgc-on. In Figure $3 d$ the boundary contours have been deleted, and in both these figures, as well as all the corresponding views of P89 and 139 , the $Z$-axis has been "exploded" $50 \times$ for clarity. These views illustrate a strength and a limitation of the present software. The strength is that measurements of the distance between a given myelin profile and the nearest point on the optic nerve meninges can and do use the vector of the $Z$-axis shown here in exaggerated form, as well as the vectors of the $X$ - and $Y$-axes 


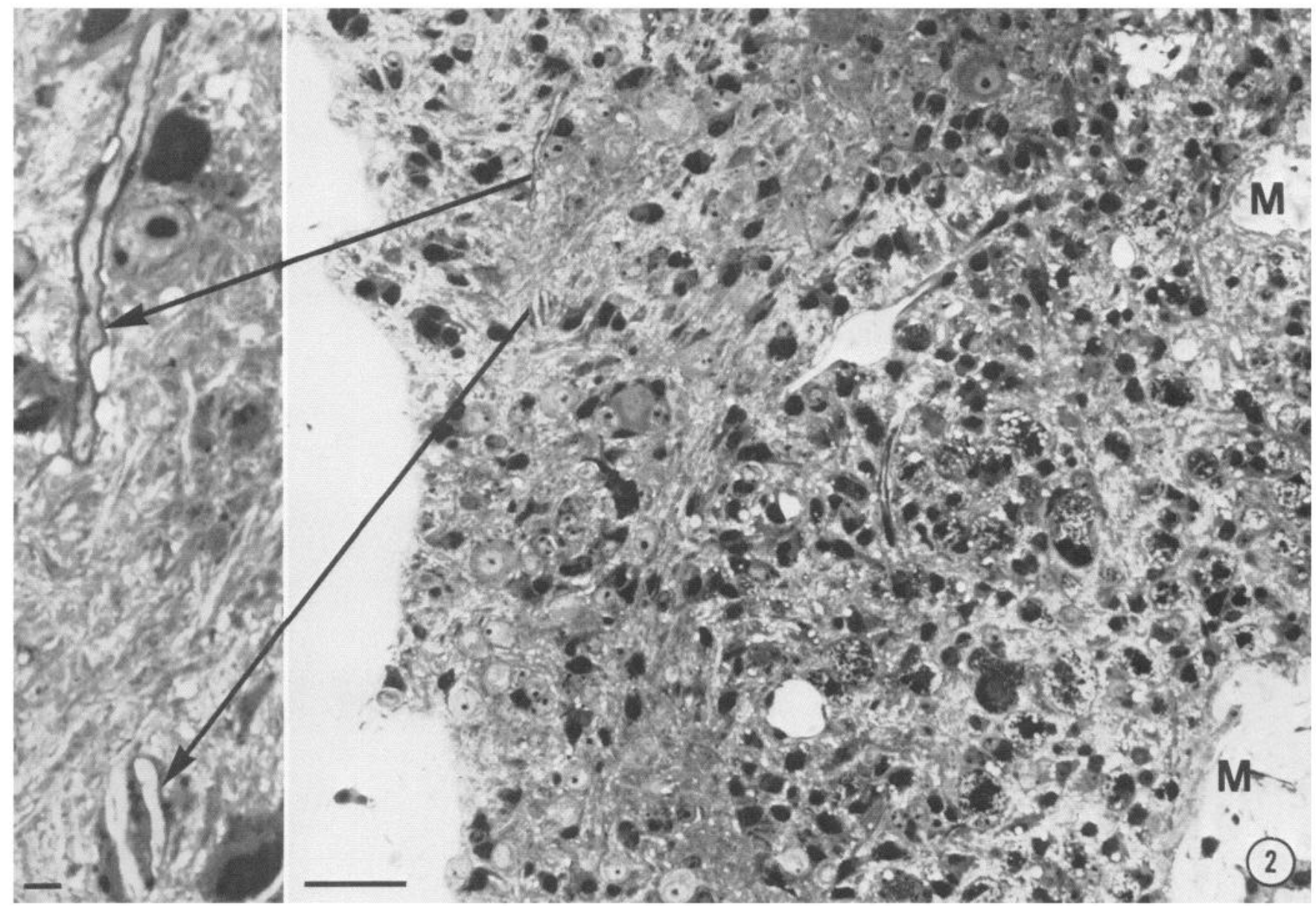

Figure 2. P139 optic nerve plus Ara-C treated cerebellum. Two portions of axons whose myelin sheaths have been made by these adult myelinating glia are shown at higher magnification to the left (arrows). While the explanted optic nerve fragments are out of the plane of section, their meninges $(M)$ are present. Bar, $50 \mu \mathrm{m}$ (right); $5 \mu \mathrm{m}$ (left).

visible in Figure 3, $a$ and $b$. The limitation is that we have no way at present to connect images of the same structure, such as a myelin sheath, seen in 2 adjacent sections. A myelin sheath that runs, for example, through 10 adjacent sections will be counted as 10 consecutive unrelated profiles. Thus, although one may form a qualitative impression that one reconstruction has more myelin than another, this cannot at present be quantitated as a number of myelin internodes or myelinated axons.

Figure $3, e$ and $f$, shows the $60^{\circ}$ and edge-on views of P89, while Figure $3, g$ and $h$, shows similar views of P139. In comparing these to the corresponding images of $\mathrm{P} 8$, recall that $\mathrm{P} 8$ contains an entire optic nerve; the meninges form a tube with 2 cut ends and additional incisions in its wall. P89 and 139 contain about $1 / 10$ of an optic nerve in 2 half-disk-shaped pieces; the meninges are confined to part of the edge of these half-disks and appear as nondescript tangles. The 2 older cultures also seem to contain a smaller number of myelin profiles than P8, although as noted above this cannot be quantitated. In P89 and 139 , as in P8, myelin begins immediately adjacent to the meninges and extends continuously to a distant point in the culture but not uniformly to all regions of the culture. In P89, the 2 fragments of optic nerve adhered by chance to different locations when explanted. It is notable that most of the myelin appears to be contiguous with the piece of nerve on the left side of the photographs. In $\mathrm{P} 139$, the $60^{\circ}$ view (Fig. $3 g$ ) suggests that the patch of myelin farthest from the optic nerve is separated by myelin-free terrain from the next nearest patch. The edge-on view (Fig. $3 h$ ) shows that, in fact, there are myelin profiles connecting this patch to the next one; all the myelin in the culture is distributed along a single pathway reaching in an S-shaped curve (as seen in the $Z$-axis view) from the optic nerve meninges to the farthest available corner of the cerebellar explant.

Figure 3. Computer-generated reconstructions of cerebellar cultures to which P8 $(a-d), \mathrm{P} 89(e, f)$, and P139 $(g, h)$ optic nerve have been added. Blue, boundary of culture; red, optic nerve meninges; green, myelin profiles. Hidden line suppression has been used, and the $Z$-axis is expanded $50 \times$ for clarity. $a$, P8 viewed from the upper surface (facing lying drop of medium). $b$, P8 viewed from the lower surface (facing collagen substrate). At P8 an entire optic nerve is explanted and is seen surrounded by its tubular sheath of meninges. Myelin profiles associated with incisures in the optic nerve meninges can be seen in $b$. A microscopic section of P8 was shown in Figure 1. $c$, P8 viewed at $60^{\circ} . d$, P8 viewed at $90^{\circ}$. In these 2 views, note the continuous distribution of myelin profiles from the optic nerve outward to the boundaries of the culture at the left. $e$, P89 viewed at $60^{\circ} . f, \mathrm{P} 89$ viewed at $90^{\circ}$. Adult optic nerve has been transplanted as 2 small half-disks. Note that most of the myelin in P89 appears to be contiguous with the piece of nerve on the left side. $g$, P139 viewed at $60^{\circ} . h, \mathrm{P} 139$ viewed at $90^{\circ}$. Here the continuity of myelin profiles with the optic nerve explant is demonstrated by tilting the culture to $90^{\circ}$, revealing a curved continuous pathway from the optic nerve meninges to the farthest available area of the cerebellar explant. The smaller number of myelin profiles in this adult culture (cf. P8) may possibly be a reflection of the smaller amount of adult optic nerve that can be successfully explanted in this system. 

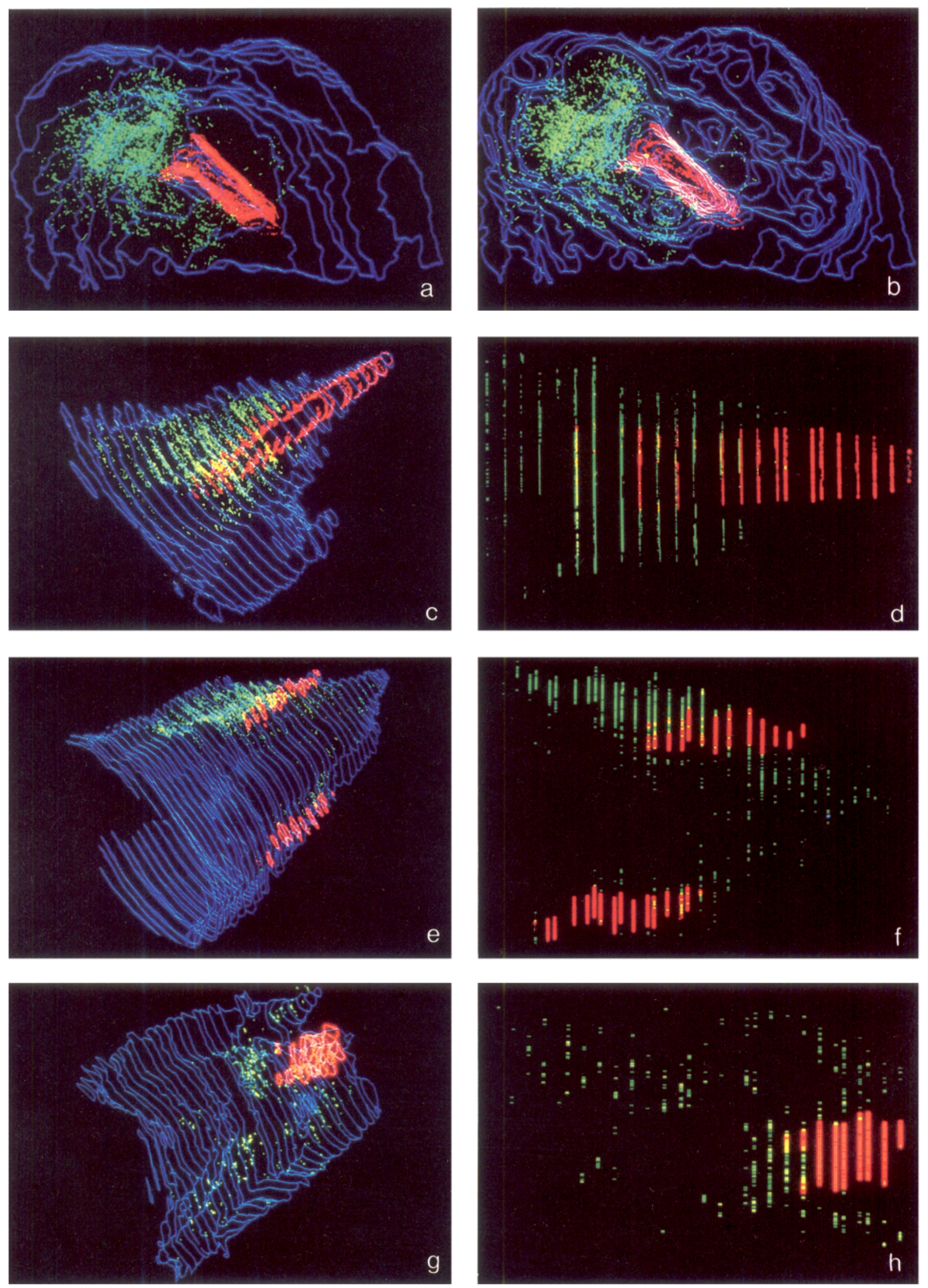
$\mathrm{P}-8$

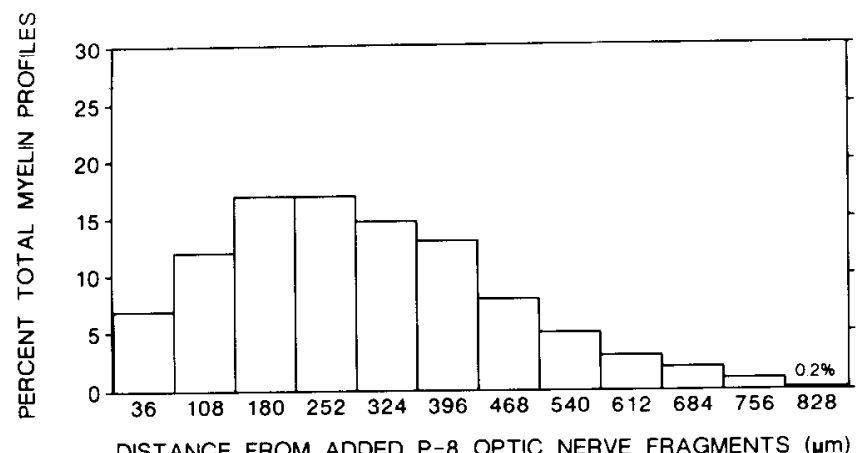

$P-89$

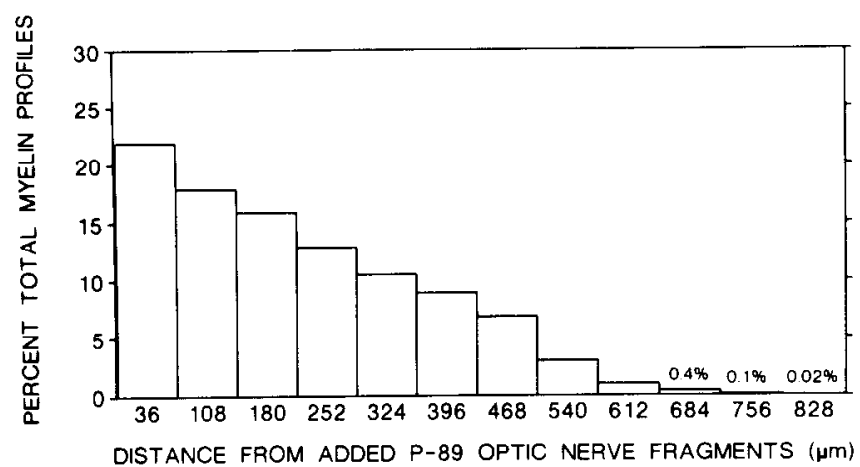

$P-139$

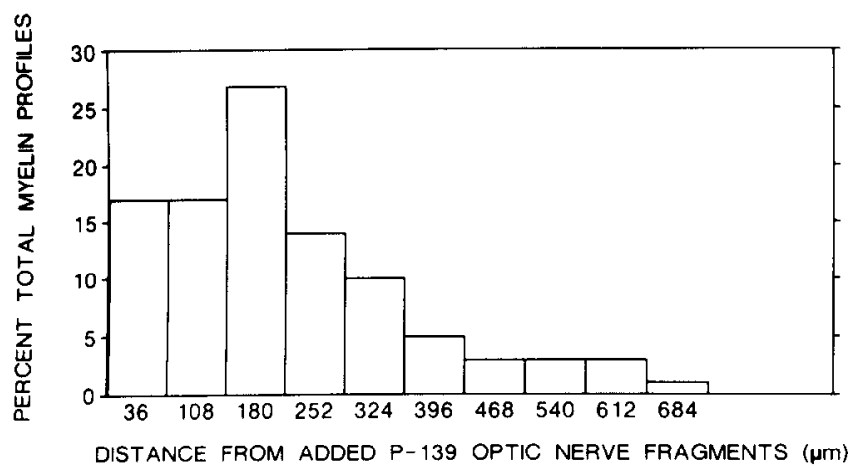

Figure 4. Histograms comparing the minimum possible migration distances of P8, 89, and 139 myelinating glia. Both immature (P8) and adult $(\mathrm{P} 89,139)$ cells can migrate more than $0.6 \mathrm{~mm}$. Actual migration distances are probably greater.

Measurements of the distances between each myelin sheath and the nearest point on the optic nerve meninges are summarized as histograms for all 3 reconstructions in Figure 4. Because of the need for a nearest-neighbor assumption, the migration trajectories estimated in this way for myelinating glial cells are shorter than the true ones. However, these numbers confirm the qualitative impression that myelinating glia from P89 or 139 nerve can migrate as far as glia from P8 nerve before forming myelin.

Successful cultures with year-old optic nerve have been obtained only recently, and reconstructions have not yet been carried out. Figure 5 shows living myelin as seen in a culture with
P376 optic nerve. It looks identical to living myelin produced by younger or even immature nerve in this culture system.

\section{Discussion}

The present experiments confirm that in this culture system new myelin is formed by glial cells present in adult mammalian CNS. There has long been evidence that regeneration of myelin in adult CNS might be more successful than elongative regeneration of axons. Clinical evidence for such regeneration has been inferred from the finding of uniformly thin, short myelin internodes intercalated between internodes of normal thickness at the edges of chronic multiple sclerosis (MS) plaques (Prineas and Connell, 1979). Experimental evidence for remyelination by glial cells in the mature CNS was reported by Bunge et al. (1961) from CSF "barbotage" lesions of the cat spinal cord and subsequently by others using various means of inducing demyelination in situ (e.g., cuprizone: Blakemore, 1974; cyanide: Hirano et al., 1968; EAE serum: Lampert, 1965; Prineas et al., 1969). Remyelination of CNS axons following experimentally induced demyelination may also occur in vitro (e.g., EAE serumtreated spinal cord cultures: Raine and Bornstein, 1970). Wood and Bunge (1986) have obtained myelin formation in an in vitro system employing dissociated adult glial cells. The unique feature of the presently described system is that the glial cells are added to the culture as a discrete block of tissue, the location of which defines the beginning of the trajectory of migration of myclinating glia. Thus, in this systcm it will be possible to monitor and manipulate the migratory process as well as the formation of myelin.

The experiments reported here do not, however, address the question of which cells in the adult optic nerve initially colonize the cerebellar explants and ultimately produce myelin. The rcsults could equally well be explained by migration of glial progenitor cells (Raff et al., 1983, 1985), nonmyelinating oligodendrocytes, or oligodendrocytes that had previously formed myelin. It is also not clear whether mitosis can or must occur as a prelude to the myelination by adult glia described here. Cell division producing additional oligodendrocytes has been reported to precede remyelination after experimental demyelination in situ (Herndon et al., 1977; Johnson and Ludwin, 1981; Ludwin, 1979) and in vitro (Raine and Bornstein, 1970), and may possibly occur in MS lesions (Raine et al., 1981). Whether these new oligodendrocytes arise primarily from division of glial progenitor cells (ffrench-Constant and Raff, 1986; Wood and Bunge, 1986) or division of mature oligodendrocytes (Arenella and Herndon, 1984; Ludwin, 1984, 1985) is presently unresolved. It is possible that future work with this culture system will provide evidence concerning the lineage and mitotic history of cells responsible for making myelin. Until it does, we confine ourselves to the neutral terminology, "myelinating glial cells," and to the important task of characterizing their behavior, whatever their lineage.

Myelin has been formed in this sytem by the oldest optic nerve glia tried: the age limit, if any, for myelin formation by adult glia remains undetermined. Cultures with adult optic nerve seem to contain smaller numbers of myelin profiles than cultures with immature nerve. This may simply reflect the constraints of the culture method. Success with adult optic nerve requires explanting a small fragment, rather than most of a nerve, as is the case with immature tissue; this means that a smaller number of glial cells are explanted in the adult. Alternatively, it is possible that adult tissue contains a smaller proportion of cells competent to regenerate myelin or that the competent cells from adult tissue undergo fewer divisions once explanted. These factors might also bear on the observation that a longer time in vitro seems to be required by adult than by immature glia to produce myelin detectable by light-microscopic examination of living cultures. Presumably, if less myelin is present in a culture, 


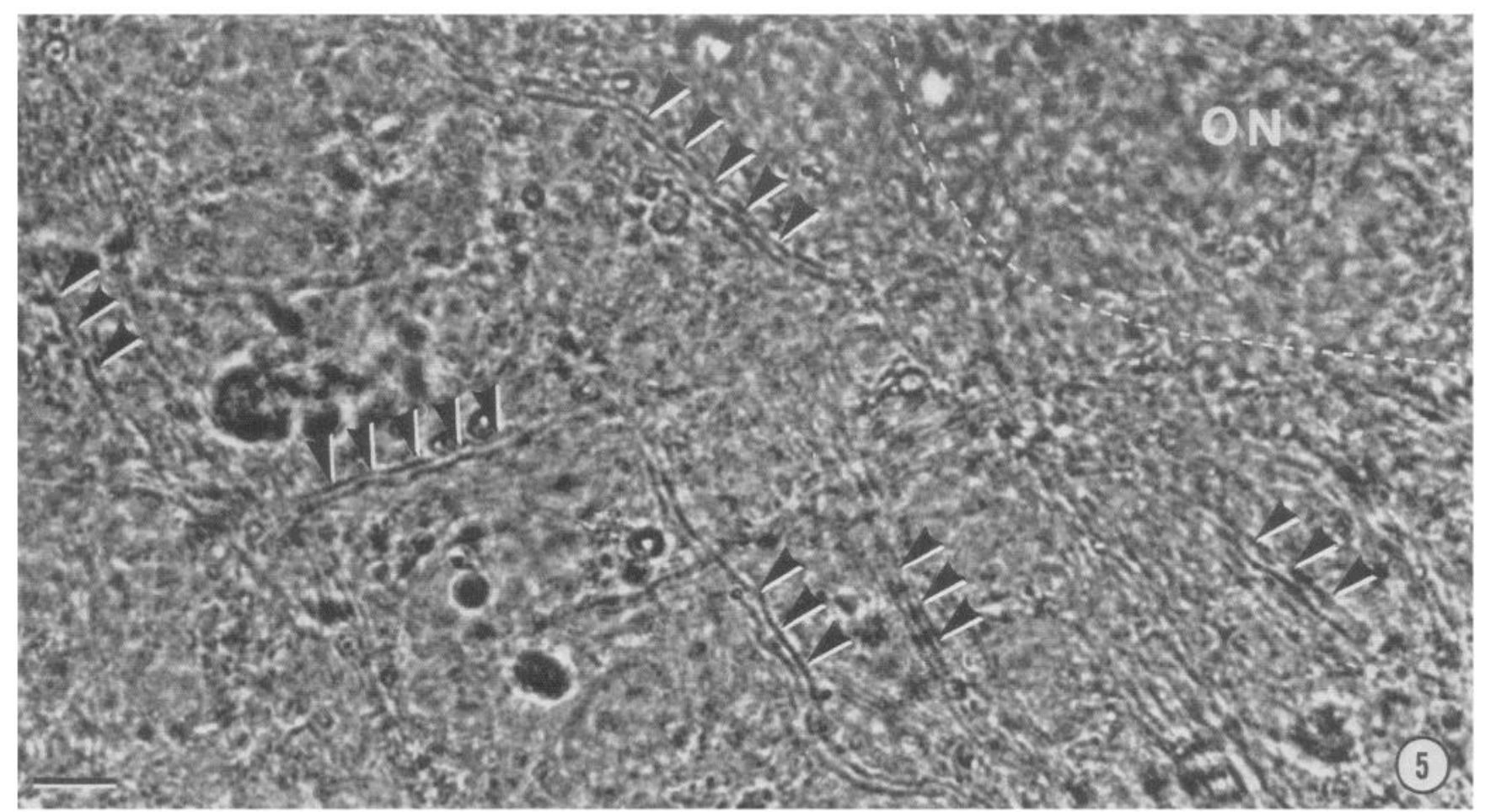

Figure 5. P376 optic nerve plus Ara-C-treated cerebellum. In this living culture, numerous axons surrounded by myelin sheaths criss-cross the field (arrowheads). The explanted adult optic nerve $(O N)$ is located at the upper right $(---)$. This living myelin, made by myelinating glia from year-old optic nerve, is indistinguishable from myelin made by immature myelinating glia. Bar, $10 \mu \mathrm{m}$.

the probability of detecting it is reduced. However, previous experience with the culture system suggests that, unless the sheaths made by regenerating adult glia are much thinner than those made by immature glia, even a few myelin sheaths should be detected. We suspect that the slower production of myelin by adult glia is a genuine difference from immature glia. This issue could be resolved by examination of fixed, sectioned cultures at serial intervals after adding either adult or immature glia.

Numerical analysis of 3-dimensional reconstructions demonstrates that myelinating glial cells of adult CNS can migrate at least $0.6 \mathrm{~mm}$ to form new myelin. Because of the nearestneighbor assumption, the true number must be larger. In fact, visual analysis strongly suggests that myelinating glial cells migrate to the most remote parts of the available axonal terrain. This is apparent at once from the $60^{\circ}$ and edge-on views of P8 and 139. In the corresponding views of P89, it appears that myelin stops short of the most remote regions of the culture; however, microscopic examination of the sections showed that these remote areas consisted entirely of astrocytic outgrowth and were unlikely to contain any potential receptive axons. Thus, we believe that the limits of migratory capacity, like the age limits for myelin formation, have not yet been determined.

A further question about glial migration is whether myelinating glial cells can migrate for any distance through neuropil devoid of receptive axons and subsequently contact such axons, or whether they must have contact guidance from receptive axons throughout their migration. Studies of the reconstructions favor the contact guidance idea, although not conclusively. For example, myelin is always found directly adjacent to the optic nerve fragment itself, and the part of the culture containing myelin always appears to be a single continuum, not 2 or more. It may be no coincidence that the success rate for these experiments, approximately $50 \%$, resembles the proportion of the cerebellar explant that contains myelin in the successful cultures. Perhaps the success rate is limited by the random chance of placing the optic nerve explant in the correct enabling relationship to the receptive axons. The distribution of myelin in P89 would seem to support this idea. Most of the myelin seems to have come from the optic nerve fragment on the left side of the photographs in Figure 3, $e$ and $f$; very little if any of it has come from the fragment on the right side. Perhaps the left fragment was more favorably located than the right one with respect to the available axonal terrain. Note that if one accepts the contact guidance idea, it is necessary to postulate an elaborately curved pathway of migration to account for a distribution of myelin such as observed in P139. This would mean that the distances over which the cells had migrated to form the myelin would be 2 or more times as large as the distances calculated under a "nearest neighbor" assumption.

A possible limitation of this culture system as a model of CNS myelin regeneration is the extent to which the successful outcome is influenced by the prior treatment of the cerebellar explants with Ara-C. This antimitotic agent, in addition to removing myelinating glial cells, kills most, though not all, granule cell neuron precursors and is also reported to delay reactive gliosis in situ (Politis and Houle, 1985). Might the looseness of Ara-C-treated neuropil promote migratory behavior by myelinating glial cells that would be unlikely to succeed in normal or gliotic neuropil? A partial rebuttal is provided by our extensive prior experience with this system in the analysis of hypomyelinated mutant disorders. Cerebellar tissue from the various mutant mice was never treated with Ara-C. All the mutant mice have astrocytosis of varying degree as a reaction of those cells to the quantitative deficiency of myelin. Presumably, this should pose obstacles to the colonization of mutant tissue by foreign normal myelinating glia. However, all 5 of the mouse mutants studied do accept in vitro colonization of mutant cerebellum by normal glia; the experiment was productively used in that way for several years before trying Ara-C treatment (Billings-Gagliardi et al., 1983, 1984; Wolf et al. 1981). In the best experiments, colonization of mutant cerebellar tissue by normal my- 
elinating glia was as extensive as in the best of the Ara-C experiments. We have done a few cultures of adult optic nerve on jimpy mutant cerebellum, and the success rate appears to be comparable to that seen with Ara-C. In future work, cultures of jimpy cerebellum will provide an important control for any possible idiosyncrasies of Ara-C treatment, although such cultures have the disadvantage that they always contain a small amount of their own myelin, formed by a few surviving jimpy oligodendrocytes.

Another limitation of the culture system to be considered is that it employs adult glial cells but immature axons. The glial cells colonize the cerebellar axons at precisely the phase of their life, in vitro or in situ, at which normal myelin formation should be proceeding. While this provides the most favorable possible setting for the myelinating glia to display all their capabilities, it fails to take account of any limitations that may be imposed on regeneration of adult myelin in situ by the abilities of adult axons to evoke or accept myelination. Since there is evidence from many different experimental systems that some regeneration of adult myelin can proceed in situ, such limitations, if they exist, cannot be absolute. A direct experimental investigation of the receptiveness of adult axons cannot be mounted at present because the preparation of organotypic cultures from adult CNS containing axons receptive to myelination is an unsolved technical problem. It should be possible, however, to let Ara-C-treated cultures of cerebellum attain an in vitro age corresponding to adulthood and then confront them with new explants of adult optic nerve.

The organotypic optic nerve/cerebellum culture system described here provides a unique opportunity to further the study of the ability of adult glia to migrate and produce new myelin. This is the cellular regenerative behavior one would hope to promote in human CNS to heal the lesions of demyelinating diseases. Experiments can be designed to determine the nutritional requirements for this regenerative behavior, which may be quite different for adult than for immature glia. The system can also be used to test factors that might promote or inhibit myelin regeneration. For example, Herndon and Triarhou (1985) recently reported that dexamethasone suppresses the demyelination that follows lysolecithin injection, but apparently at the cost of delaying remyelination, while azathioprine and cyclophosphamide enhance remyelination. The in vitro system may become important in future evaluation of these and other therapeutic interventions for demyelinating diseases.

\section{References}

Arenella, L. S., and R. M. Herndon (1984) Mature oligodendrocytes. Division following experimental demyelination in adult animals. Arch. Neurol. 41: 1162-1165.

Billings-Gagliardi, S., L. H. Adcock, E. D. Lamperti, G. Schwing-Stanhopc, and M. K. Wolf (1983) Myclination of jp, jp ${ }^{\text {msd }}$, and qk axons by normal glia in vitro: Ultrastructural and autoradiographic evidence. Brain Res. 268: 255-266.

Billings-Gagliardi, S., A. L. Hall, G. B. Stanhope, R. J. Altschuler, and M. K. Wolf (1984) Cultures of shiverer mutant cerebellum injected with normal oligodendrocytes make both normal and shiverer myelin. Proc. Natl. Acad. Sci. USA 81: 2558-2561.

Blakemore, W. F. (1974) Remyelination of the superior cerebellar peduncle in old mice following demyelination induced by cuprizone. J. Neurol. Sci. 22: 121-126.

Bunge, M. B., R. P. Bunge, and H. Ris (1961) Ultrastructural study of remyelination in an experimental lesion in adult cat spinal cord. J. Biophys. Biochem. Cytol. 10: 67-94.

ffrench-Constant, C., and M. C. Raff (1986) Proliferating bipotential glial progenitor cells in adult rat optic nerve. Nature 319:499-502.

Herndon, R. M., and L. C. Triarhou (1985) The effect of immunosuppressive drugs on remyelination. Soc. Neurosci. Abstr. 11: 422.

Herndon, R. M., D. L. Price, and L. P. Weiner (1977) Regeneration of oligodendroglia during recovery from demyelinating disease. Science 195: 693-694.
Hirano, A., S. Levine, and H. M. Zimmerman (1968) Remyelination in the central nervous system after cyanide intoxication. J. Neuropathol. Exp. Neurol. 27: 234-245.

Johnson, E. S., and S. K. Ludwin (1981) The demonstration of recurrent demyelination and remyelination of axons in the central nervous system. Acta Neuropathol. (Berl.) 53: 93-98.

Kelton, D. E., and H. Rauch (1962) Myelination and myelin degeneration in the central nervous system of dilute-lethal mice. Exp. Neurol. 6: 252-262.

Lachapelle, F., M. Gumpel, M. Baulac, C. Jacque, P. Duc, and N. Baumann (1984) Transplantation of CNS fragments into the brain of shiverer mutant mice: Extensive myelination by implanted oligodendrocytes. I. Immunohistochemical studies. Dev. Neurosci. 6 . 325-334.

Lampert, P. W. (1965) Demyelination and remyelination in experimental allergic encephalomyclitis. Further elcetron microscopic observations. J. Neuropathol. Exp. Neurol. 24: 371-385.

Ludwin, S. K. (1979) An autoradiographic study of cellular proliferation in remyelination of the central nervous system. Am. J. Pathol. 95: 683-696.

Ludwin, S. K. (1984) Proliferation of mature oligodendrocytes after trauma to the central nervous system. Nature 308: 274-275.

Ludwin, S. K. (1985) Reaction of oligodendrocytes and astrocytes to trauma and implantation. A combined autoradiographic and immunohistochemical study. Lab. Invest. 52: 20-30.

Pearlstein, R. A., L. Kirschner, J. Simons, S. MacKell, W. F. White, and R. L. Sidman (1986) A multimodal system for reconstruction and quantification of neurological structure. Anal. Quant. Cytol. (in press).

Politis, M. J., and J. D. Houlc (1985) Effect of cytosinc arabinofuranoside (Ara C) on reactive gliosis in vivo. An immunohistochemical and morphometric study. Brain Res. 328: 291-300.

Prineas, J. W., and F. Connell (1979) Remyelination in multiple sclerosis. Ann. Neurol. 5: 22-31.

Prineas, J., C. S. Raine, and H. Wisniewski (1969) An ultrastructural study of experimental demyelination and remyelination. III. Chronic experimental allergic encephalomyelitis in the central nervous system. Lab. Invest. 21: 472-483.

Raff, M. C., E. R. Abney, and J. Fok-Seang (1985) Reconstitution of a developmental clock in vitro: A critical role for astrocytes in the timing of oligodendrocyte differentiation. Cell 42: 61-69.

Raff, M. C., R. H. Miller, and M. Noble (1983) Glial cell lineages in the rat optic nerve. Cold Spring Harbor Symp. Quant. Biol. 48: 569572.

Raine, C. S., and M. B. Bornstein (1970) Experimental allergic encephalomyelitis: A light and electron microscope study of remyelination and "sclerosis" in vitro. J. Neuropathol. Exp. Neurol. 29: 552574.

Raine, C. S., L. Scheinberg, and J. M. Waltz (1981) Multiple sclerosis. Oligodendrocyte survival and proliferation in an active established lesion. Lab. Invest. 45: 534-546.

Schwing-Stanhope, G. B., and M. K. Wolf (1983) Methodological appendix: A tissue culture strategy for studying mutant mice with defective CNS myelination. In Neuroscience Approached Through Cell Culture, Vol. 2, S. E. Pfeiffer, ed., pp. 155-165, CRC Press, Boca Raton, FL.

Seil, F. J., A. L. Leiman, and W. R. Woodward (1980) Cytosine arabinoside effects on developing cerebellum in tissue culture. Brain Res. 186: 393-408.

Skoff, R. P., D. L. Price, and A. Stocks (1976) Electron microscopic autoradiographic studies of gliogenesis in rat optic nerve. II. Time of origin. J. Comp. Neurol. 169: 313-334.

Stanhope, G. B., M. K. Wolf, and S. Billings-Gagliardi (1986) Genotype-specific myelin formation around normal axons in cytosine arabinoside-treated organotypic cultures injected with normal or shiverer optic nerve. Dev. Brain Res. 24: 109-116.

Wolf, M. K., M. Brandenberg, and S. Billings-Gagliardi (1985) Evidence for remyelinative potential of adult oligodendrocytes: Computer reconstructive analysis of tissue culture experiments. Soc. Neurosci. Abstr. 11: 422 .

Wolf, M. K., G. B. Schwing, L. H. Adcock, and S. Billings-Gagliardi (1981) Hypomyclinated mutant mice. III: Increased myelination in mutant cerebellum co-cultured with normal optic nerve. Brain Res. 206: 193-197.

Wood, P. M., and R. P. Bunge (1986) Evidence that axons are mitogenic for oligodendrocytes isolated from adult animals. Nature 320 : $756-758$ 